International Journal of Pure and Applied Mathematics

Volume 87 No. $6 \quad 2013$, 763-769

ISSN: 1311-8080 (printed version); ISSN: 1314-3395 (on-line version)

url: http://www.ijpam.eu

doi: http://dx.doi.org/10.12732/ijpam.v87i6.5

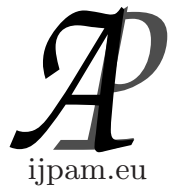

\title{
SUPER STRONGLY PERFECTNESS OF SOME GRAPHS
}

\author{
R. Mary Jeya Jothi ${ }^{1}$, A. Amutha ${ }^{2}$ \\ ${ }^{1,2}$ Department of Mathematics \\ Sathyabama University \\ Chennai, 119, INDIA
}

\begin{abstract}
A Graph $G$ is Super Strongly Perfect Graph if every induced sub graph $H$ of $G$ possesses a minimal dominating set that meets all the maximal cliques of $H$. The structure of Super Strongly Perfect Graphs have been characterized by some classes of graphs like Cycle graphs, Circulant graphs, Complete graphs, Complete Bipartite graphs etc., In this paper, we have analysed some other graph classes like, Bicyclic graphs, Dumb bell graphs and Star graphs to characterize the structure of Super Strongly Perfect Graphs in a different way. By this we found the cardinality of minimal dominating set and maximal cliques of the above graphs.
\end{abstract}

AMS Subject Classification: $05 \mathrm{C} 75$

Key Words: super strongly perfect graph, minimal dominating set, bicyclic graph, dumb bell graph

\section{Introduction}

In this paper, graphs are finite and simple, that is, they have no loops or multiple edges. Let $G=(V, E)$ be a graph. A clique in $G$ is a set $X \subseteq V(G)$ of pair wise adjacent vertices. A subset $D$ of $V(G)$ is called a dominating set if every vertex in $V-D$ is adjacent to at least one vertex in $D$. A subset $S$ of $V$ is said to be a minimal dominating set if $S-\{u\}$ is not a dominating set for

Received: September 6, 2013

(c) 2013 Academic Publications, Ltd. url: www.acadpubl.eu 
any $u \in S$. A complete graph is a simple undirected graph in which every pair of distinct vertices is connected by a unique edge. The complete graph on $n$ vertices is denoted by $K_{n}$. A complete bipartite graph, $G=\left(V_{1}+V_{2}, E\right)$, is a bipartite graph such that for any two vertices, $v_{1} \in V_{1}$ and $v_{2} \in V_{2}, v_{1} v_{2}$ is an edge in $G$. The complete bipartite graph with partitions of size $\left|V_{1}\right|=m$ and $\left|V_{2}\right|=n$, is denoted $K_{m, n}$. A path in a graph is a sequence of vertices such that from each of its vertices there is an edge to the next vertex in the sequence. A closed path is called a cycle and it is denoted by $C_{n}$. The number of vertices in $C_{n}$ equals the number of edges. The cycle graph with even number of vertices is called an even cycle and the cycle graph with odd number of vertices is called an odd cycle. A tree is a connected acyclic graph. A Wheel Graph $G=(V, E)$ be a graph with $n$ vertices formed by connecting a single vertex to all vertices of an $(n-1)$ cycle. It is denoted by $W_{n}$.

\section{Overview of the Paper}

We have characterized the structure of Super Strongly Perfect Graphs in some classes of graphs like Cycle graphs, Circulant graphs, Complete graphs, Complete Bipartite graphs etc., $[1,2,4]$. In this paper, we have discussed some other graph classes like, Bicyclic graphs, Dumb bell graphs and Star graphs to characterize the structure of Super Strongly Perfect Graphs. By this investigation, we have found the cardinality of minimal dominating set and maximal cliques of the above graphs.

\subsection{Super Strongly Perfect Graph (SSP)}

A Graph $G=(V, E)$ is Super Strongly Perfect if every induced sub graph $H$ of $G$ possesses a minimal dominating set that meets all the maximal cliques of $H$. Every Super Strongly Perfect graph with maximal complete sub graph $K_{2}$ is isomorphic to any bipartite graph. Any arbitrary graph which does not contain an odd cycle of length at least five is isomorphic to every Super Strongly Perfect graph with maximal complete sub graph $K_{n}$ where $n \geq 3$ [2].

Example 1. In Figure 1, $\{1,5\}$ is a minimal dominating set which meets all maximal cliques of $G$.

Example 2. In Figure 2, $\{1,2,5,8\}$ is a minimal dominating set which does not meet all maximal complete sub graphs $K_{2}$. Also we cannot find a minimal dominating set which meets all maximal complete sub graphs $K_{2}$. Hence the graph is non-Super Strongly Perfect. 


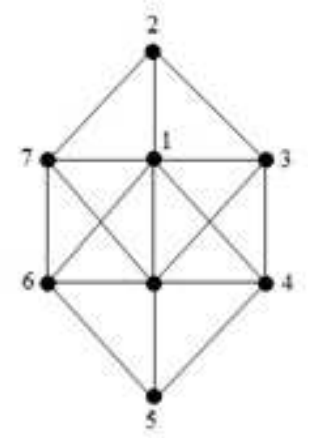

Figure 1: Super Strongly Perfect Graph

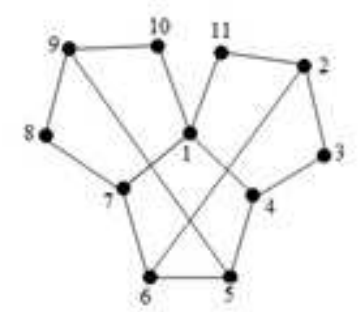

Figure 2: Non-Super Strongly Perfect Graph

\section{Bicyclic Graph}

A Bicyclic graph $D_{a, b, c}$ is a graph consisting of two vertex disjoint cycles $C_{a+1}$, $C_{b+1}$ and a Path $c$ joining them having only its end-vertices in common with the two cycles. Also a connected $(p, p+1)$ graph is called a Bicyclic graph. Bicyclic graphs are connected graphs with two independent cycles. Refer the example in Figure 3.

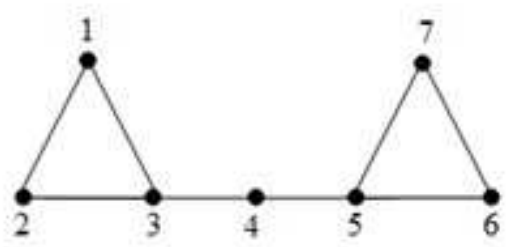

Figure 3: $D_{2,2,3}$ 
Theorem 1. Every Bicyclic graph $D_{a, b, c}$ where $a=2, b \geq 3, b$ is odd (or) $b=2, a \geq 3, a$ is odd, is Super Strongly Perfect Graph.

Proof. Let $G$ be a Bicyclic graph $D_{a, b, c}$ where $a=2, b \geq 3, b$ is odd (or) $b=2, a \geq 3, a$ is odd.

$\Rightarrow G$ is bipartite.

$\Rightarrow G$ is Super Strongly Perfect.

Hence every Bicyclic graph $D_{a, b, c}$ where $a=2, b \geq 3, b$ is odd (or) $b=2$, $a \geq 3, a$ is odd, is Super Strongly Perfect.

Theorem 2. Every Bicyclic graph $D_{a, b, c}$ where $a, b$ are odd, $a, b>2$, is Super Strongly Perfect Graph.

Proof. Let $G$ be a Bicyclic graph $D_{a, b, c}$ where $a, b$ are odd.

$\Rightarrow G$ is bipartite.

$\Rightarrow G$ is Super Strongly Perfect.

Hence Bicyclic graph $D_{a, b, c}$ where $a, b$ are odd is Super Strongly Perfect.

Theorem 3. Every Bicyclic graph $D_{a, b, c}$ where $a, b$ are even (or) $a$ is odd and $b$ is even (or) $a$ is even and $b$ is odd, is non-Super Strongly Perfect Graph.

Proof. Let $G$ be a Bicyclic graph $D_{a, b, c}, a, b>2$, where $a, b$ are even (or) $a$ is odd and $b$ is even (or) $a$ is even and $b$ is odd.

$\Rightarrow G$ is non-bipartite with maximal complete sub graph $K_{2}$.

$\Rightarrow G$ is non-Super Strongly Perfect.

Hence every Bicyclic graph $D_{a, b, c}$ where $a, b$ are even (or) $a$ is odd and $b$ is even (or) $a$ is even and $b$ is odd, is Super Strongly Perfect.

Proposition 1. Every $D_{a, b, c}$ where $a=2, b \geq 3, b$ is odd (or) $b=2, a \geq 3$, $a$ is odd, which is Super Strongly Perfect Graph has the following properties:

1) It contains one maximal complete sub graphs on 3 vertices.

2) It contains a minimal dominating set of cardinality $\left\lfloor\frac{a+b+c}{2}\right\rfloor-1$.

3) It is 3-colourable.

Proposition 2. Every Bicyclic graph $D_{a, b, c}$ where $a, b$ are odd, $a, b>2$, which is Super Strongly Perfect Graph has the following properties:

1) It contains $a+b+c+1$ maximal complete sub graphs on 2 vertices.

2) It contains a minimal dominating set of cardinality $\left\lceil\frac{a+b+c}{2}\right\rceil-1$. 
3) It is 2-colourable.

\section{Dumb Bell Graph}

A Dumb bell graph $D_{r, s, t}$ is a graph which is obtained by taking the path graph $P_{t}$ and adding $r+1$ vertices such that the first $r$ additional vertices form a clique of order $r+1$ with the left end vertex of $P_{t}$ and the remaining $s$ vertices form a clique of order $s+1$ with the right end vertex of $P_{t}$. Hence a dumbbell graph is a simple graph obtained by joining two cliques $K_{l}$ and $K_{m}$ where $l, m \geq 2$, with a path of length $i, i \geq 2$. Dumb bell graphs have $r+s+t$ vertices and $\frac{r^{2}+s^{2}+r+s}{2}$ edges. Bicyclic graphs are particular case of dumb bell graphs $D_{r, s, t}$, $r=s=2, t \geq 2$.

\section{Example 3.}

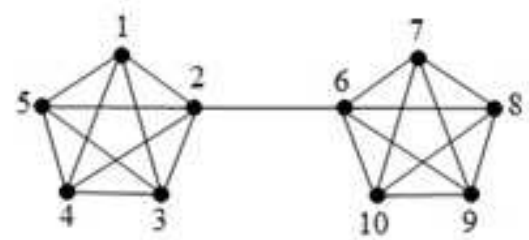

Figure 4: $D_{4,4,2}$

Theorem 1. Every Dumb bell graph $D_{r, s, t}$, is Super Strongly Perfect Graph.

Proof. Let $G$ be a Dumb bell graph.

$\Rightarrow G$ does not contain an odd cycle of length at least five as an induced sub graph.

$\Rightarrow G$ is Super Strongly Perfect.

Hence every dumb bell graph is Super Strongly Perfect.

Proposition 1. Every Dumb bell graph $D_{r, s, t}$ which is Super Strongly Perfect Graph has the following properties:

1) It contains one maximal complete sub graphs on $r+1$ vertices if $r>s$.

2) It contains one maximal complete sub graphs on $s+1$ vertices if $s>r$. 
3) It contains one maximal complete sub graphs on $r+1$ vertices if $r=s$.

4) It is $r+1$ colourable if $r>s$.

5) It is $s+1$ colourable if $s>r$.

6) It is $r+1$ colourable if $r=s$.

7) It contains a minimal dominating set of cardinality $2+\left\lceil\frac{t-2}{2}\right\rceil$ vertices.

\section{Star Graph}

A Star $S_{k}$ is the complete bipartite graph $K_{1, k}$. It is a tree with one internal vertex and $k$ leaves (but, no internal vertices and $k+1$ leaves when $k \leq 1$ ). A star with 3 edges is called a claw. Clearly, every Star graph contains a single vertex which is adjacent to all the remaining vertices. The set of distances between the vertices of a claw provides an example of a finite metric space that cannot be embedded isometrically into a Euclidean space of any dimension [3]. The star network, a computer network modeled after the star graph, is important in distributed computing.

\section{Example 4.}

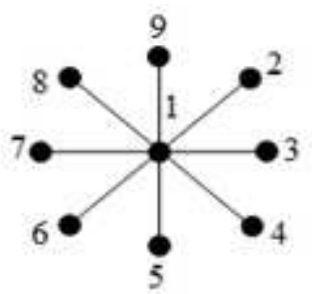

Figure 5: $S_{8}$

Theorem 1. Every Star is Super Strongly Perfect.

Proof. Let $G$ be a Star graph.

$\Rightarrow$ Let $S=\{v\}$ be the minimal dominating set of cardinality 1 .

$\Rightarrow v$ is adjacent to all the remaining vertices of $G$.

$\Rightarrow v$ meets all maximal complete sub graphs of $G$.

$\Rightarrow G$ is Super Strongly Perfect.

Hence every Star graph is Super Strongly Perfect. 
Proposition 1. Every Star graph $S_{k}$ which is Super Strongly Perfect has $k$ maximal complete sub graphs on 2 vertices.

Proposition 2. Every Star graph which is Super Strongly Perfect is 2colourable.

\section{Conclusion}

We have discussed the structural properties of Bicyclic graphs, Dumb bell graphs and Star graphs. In future this investigation is more considering for the remaining graph classes.

\section{References}

[1] Amutha. A, Mary Jeya Jothi. R, "Characterization of Super Strongly Perfect Graphs in Bipartite Graphs", Proceedings of an International Conference on Mathematical Modelling and Scientific Computation, vol.1, pp. 183-185, 2012.

[2] Amutha. A, Mary Jeya Jothi. R, "On Super Strongly Perfect Graph", International Journal of Computers, Mathematical Sciences and Applications, vol. 4, No's 1-2, pp. 195-203, 2010.

[3] Linial, Nathan, "Finite metric spaces-combinatorics, geometry and algorithms", Proc. International Congress of Mathematicians, Beijing, vol. 3, pp. 573-586, 2002.

[4] Mary Jeya Jothi. R, Amutha. A, "An introduction to the family members of the architecture Super Strongly Perfect Graph (SSP)", IEEE Xplore, pp. 1087-1091, May 2011. 
